\title{
Plasma skimming efficiency of human blood in the spiral groove bearing of a centrifugal blood pump
}

\author{
Daisuke Sakota $^{1}\left(\mathbb{0} \cdot\right.$ Kazuki Kondo $^{1} \cdot$ Ryo Kosaka $^{1} \cdot$ Masahiro Nishida $^{1} \cdot$ Osamu Maruyama $^{1}$
}

Received: 19 May 2020 / Accepted: 13 October 2020 / Published online: 28 October 2020

(c) The Author(s) 2020

\begin{abstract}
This work investigates the plasma skimming effect in a spiral groove bearing within a hydrodynamically levitated centrifugal blood pump when working with human blood having a hematocrit value from 0 to $40 \%$. The present study assessed the evaluation based on a method that clarified the limitations associated with such assessments. Human blood was circulated in a closed-loop circuit via a pump operating at $4000 \mathrm{rpm}$ at a flow rate of $5 \mathrm{~L} / \mathrm{min}$. Red blood cells flowing through a ridge area of the bearing were directly observed using a high-speed microscope. The hematocrit value in the ridge area was calculated using the mean corpuscular volume, the bearing gap, the cross-sectional area of a red blood cell, and the occupancy of red blood cells. The latter value was obtained from photographic images by dividing the number of pixels showing red blood cells in the evaluation area by the total number of pixels in this area. The plasma skimming efficiency was calculated as the extent to which the hematocrit of the working blood was reduced in the ridge area. For the hematocrit in the circuit from 0 to $40 \%$, the plasma skimming efficiency was approximately $90 \%$, meaning that the hematocrit in the ridge area became $10 \%$ as compared to that in the circuit. For a hematocrit of $20 \%$ and over, red blood cells almost completely occupied the ridge. Thus, a valid assessment of plasma skimming was only possible when the hematocrit was less than $20 \%$.
\end{abstract}

Keywords Plasma skimming $\cdot$ Hydrodynamic bearing $\cdot$ Centrifugal blood pump $\cdot$ Hemolysis $\cdot$ Optics of blood

\section{Introduction}

Understanding the blood flow dynamics in mechanical circulatory support devices is important for the development of more hemocompatible devices, with the aim of preventing thrombosis and hemolysis. Consequently, many researchers have investigated and simulated such dynamics using computational fluid dynamics (CFD) and particle image velocimetry [1-5]. However, these conventional studies have assessed blood flow solely on the macroscopic level.

Electronic supplementary material The online version of this article (https://doi.org/10.1007/s10047-020-01221-9) contains supplementary material, which is available to authorized users.

Daisuke Sakota

sakota.ao@aist.go.jp

1 Artificial Organ Research Group, Health and Medical Research Institute, National Institute of Advanced Industrial Science and Technology (AIST), 1-2-1 Namiki, Tsukuba, Ibaraki 305-8564, Japan
In fact, it is challenging to precisely simulate or even to directly visualize localized bearing areas in a rotary blood pump, which are likely the primary regions associated with the destruction of blood cells. As such, it would be helpful to have a better understanding of blood flow dynamics on the scale of individual cells at such hydrodynamic bearing regions, to assist in the development of novel hemocompatible devices.

The phenomenon of plasma skimming (PS) is observed in microvessels. This effect is defined as a reduction in the hematocrit (HCT) value in a branched vessel as compared to that in a main vessel due to the lower migration of red blood cells (RBCs) into the branch. PS was first described by Krogh [6] and has typically been investigated by observations during in vivo microcirculation or with in vitro microfluidic devices [7-11], although some researchers have also utilized artificial organs. In 1985, Lewandowski and Nose et al. reported that a PS layer is formed at membrane surfaces and speculated that controlling this effect may assist in the further development of hemofiltration [12]. Also, in 2004, Kink and Reul applied a spiral groove bearing (SGB) 
to an axial flow blood pump for the first time and developed a system in which the entry of RBCs into the bearing gap is inhibited based on the PS effect, such that hemolysis does not occur [13]. Several other studies of the PS effect in conjunction with hydrodynamic bearings have been performed but did not involve the direct observation and quantification of the degree of this phenomenon [14-16]. Although concepts to either utilize or control the PS effect have been established in past research, few quantitative evaluations have been performed. In 2016, our own group reported a method for the quantitative assessment of the PS effect in a hydrodynamically levitated centrifugal blood pump [17]. This work involved the first-ever direct quantitative analysis of PS in an SGB, using bovine blood at an HCT of $1 \%$.

Herein, we demonstrate the quantitative assessment of the PS effect in an SGB, working with human blood, whose the HCT range from 0 to $40 \%$. The maximum limitation of the evaluation of PS effect for HCT is clarified. Both the evaluation method and the theoretical basis of PS are examined and the limitations of this technique are discussed.

\section{Materials and methods}

\section{Ethical approval}

Human whole blood used in the study was donated by the Japanese Red Cross Society. The study was approved by the Life Science Experiment Application Committee at the National Institute of Advanced Industrial Science and Technology (Approved Number: hi 2017-225), Japan.

\section{Experimental setup}

The PS evaluation system was constructed as described previously [17], and is pictured in Fig. 1a. This apparatus incorporated a hydrodynamically levitated centrifugal blood pump developed by our group, the structure of which has been reported in detail in a prior publication [18]. The rotational speed of the pump was maintained at $4000 \mathrm{rpm}$ throughout the experiments, with a flow rate of $5 \mathrm{~L} / \mathrm{min}$. The flow circuit comprised a reservoir, polyvinyl chloride tubing with an inner diameter of $3 / 8$ in., a sampling port, an adjustable resistor and the blood pump. The blood temperature was maintained at $37{ }^{\circ} \mathrm{C}$. The pump components were made of transparent polymethyl methacrylate. The
Fig. 1 Experimental setup. a Photographic image of the plasma skimming evaluation system. b Diagram showing the observation point at a single groove and ridge in the spiral groove bearing of the hydrodynamically levitated centrifugal blood pump. c Microscopic image obtained using a highspeed camera. The ridge area surrounded by the dotted line is the region in which plasma skimming was evaluated. d Cross-sectional view of the observation point. Here, $\mathrm{dx}$ is the bottom bearing gap (a)

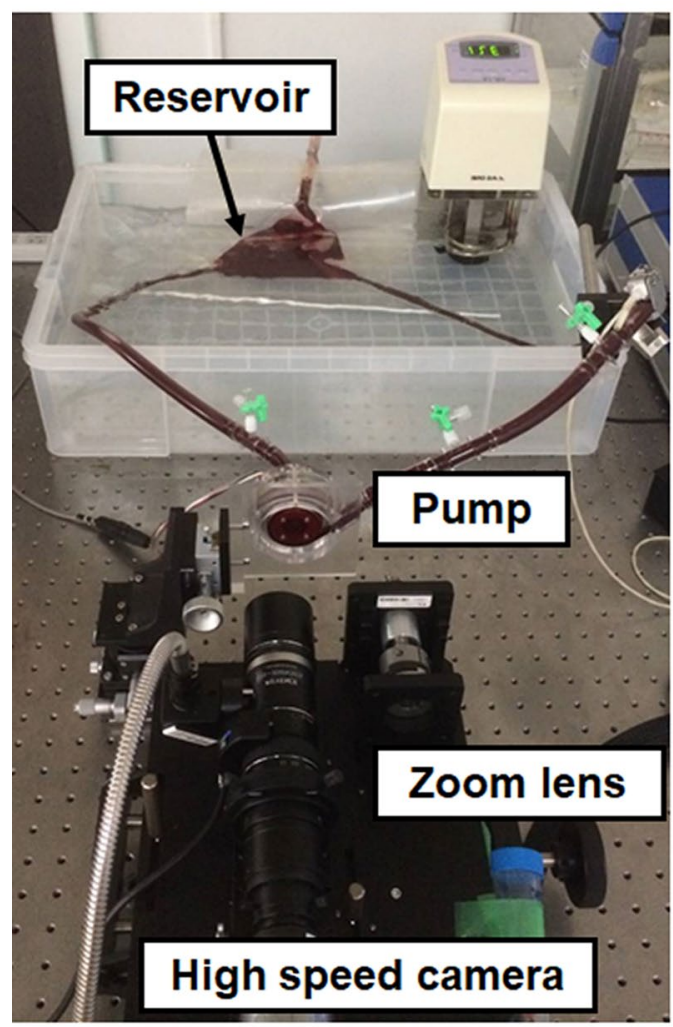

(b)

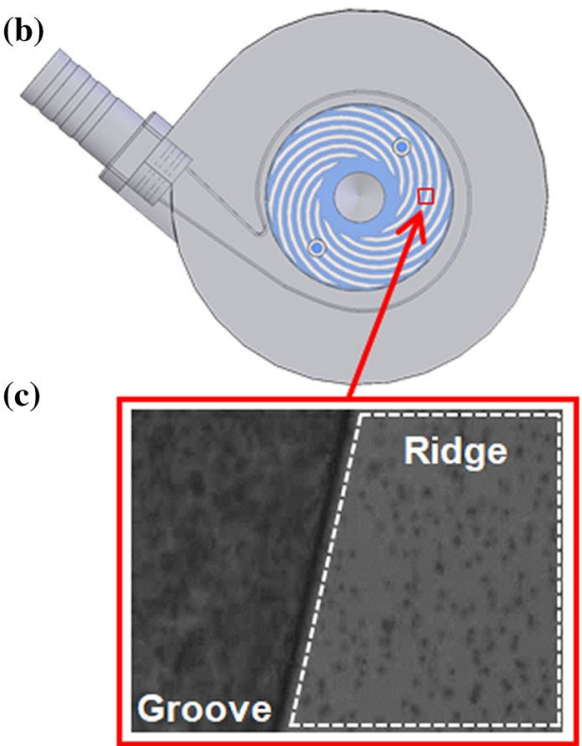

(d)

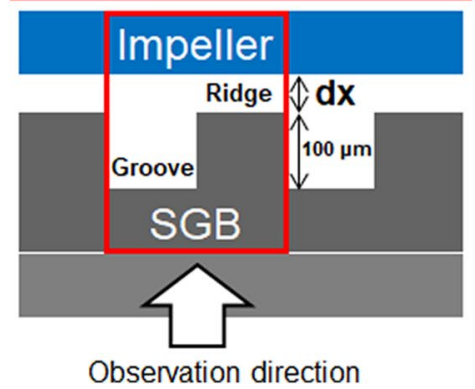


closed-type impeller had six vanes, a diameter of $37 \mathrm{~mm}$, and a height of $26 \mathrm{~mm}$, and was levitated by hydrodynamic bearings acting in the radial and thrust directions. SGBs were attached to the top and bottom casing to suspend the impeller in the thrust direction, and the bottom part between the impeller and the SGB was monitored using a high-speed camera (VW-9000; Keyence Corp., Osaka, Japan) with a long-distance high-performance zoom lens (VH-Z50W; Keyence Corp.). The shutter speed and frame rate were set to $1 / 900,000 \mathrm{~s}$ and 15,000 frames/s respectively, and a xenon lamp (MAX-303; Asahi Spectra Corp., Tokyo, Japan) was attached to the zoom lens as a light source. The camera was set up so that a single groove and ridge could be observed, as shown in Fig. 1b, and an actual image obtained from the high-speed camera is shown in Fig. 1c. Figure 1d presents a cross-sectional view of the observation point. The ridge area at this point represents the region at which the highest shear rate appeared in the pump. The shear rate was estimated to be approximately $10^{5} 1 / \mathrm{s}$ or higher at $4000 \mathrm{rpm}$. The region labeled " $\mathrm{dx}$ " in this figure represents the bottom bearing gap or the levitation distance, which varied with the hydrodynamic force generated by the SGB.

In the present experiments, the PS effect was investigated over the range of HCT values from approximately $0-40 \%$ by adding phosphate buffered saline (PBS) to the circuit. At each HCT, the ridge area was imaged by the camera over three rotational phases of the impeller and each trial was repeated five times.

\section{Plasma skimming efficiency}

In this study, the PS efficiency (PSE) was defined as follows:

$\mathrm{PSE}=1-\frac{\mathrm{HCT}_{\mathrm{R}}}{\mathrm{HCT}_{\mathrm{W}}}$

where $\mathrm{HCT}_{\mathrm{W}}$ is the hematocrit value of working or circulating blood in the circuit and $\mathrm{HCT}_{\mathrm{R}}$ is the hematocrit in the ridge area under observation. At a PSE value of $100 \%$, $\mathrm{HCT}_{\mathrm{R}}$ will be $0 \%$, meaning that all RBCs escape from the highest shear-stress area in the ridge to the lower shear-stress area in the groove. $\mathrm{HCT}_{\mathrm{W}}$ values were determined based on the blood aliquots taken from the sampling port located in the circuit tube. The calculation of $\mathrm{HCT}_{\mathrm{R}}$ has been described in a previous paper [17], and is based on assessing the optical scattering coefficient of the blood. This coefficient is calculated assuming that the RBCs are uniformly spread throughout the ridge, using the following equation [19-24]:

$\mu_{\mathrm{s}}=\frac{\mathrm{HCT}_{\mathrm{R}}}{\mathrm{MCV}} \sigma$,

where $\mu_{\mathrm{s}}$ is the scattering coefficient (with units of $1 / \mu \mathrm{m}$ ), $\mathrm{MCV}$ is the mean corpuscular volume of the RBCs and $\sigma$ is the scattering cross-section. Equation (2) indicates that a photon travelling through blood will definitely hit an RBC within a distance of $1 / \mu_{\mathrm{s}}$. As such, if the bottom bearing gap is $\mathrm{dx}=1 / \mu_{\mathrm{s}}$ at $\mathrm{HCT}_{\mathrm{R}}$, the two-dimensional ridge area shown in Fig. 1c will be occupied by RBCs. Therefore, we can write the following relation:

$\mu_{\mathrm{s}}=Q / d x$,

where $Q$ is the occupancy of RBCs in the ridge area. The value of $Q$ is obtained by dividing the number of pixels containing RBCs in the evaluation area by the total number of pixels in this region [17]. Based on Eqs. (2) and (3), $\mathrm{HCT}_{\mathrm{R}}$ can be calculated as follows:

$\mathrm{HCT}_{\mathrm{R}}=\frac{Q \times \mathrm{MCV}}{d x \times \sigma}$.

The MCV can be determined using an automated blood cell analyzer (Celltac $\alpha$ MEK-6450, Nihon Kohden Corp., Tokyo, Japan). Sakota and Takatani showed that $\sigma$ can be regarded as equivalent to the geometrical cross-section of a biconcave model RBC when working with visible or nearinfrared light [24], and can be calculated as follows:

$\sigma=\pi\left(\frac{3 \mathrm{MCV}}{4 \pi C}\right)^{2 / 3}$.

where $C(=0.39023)$ is the ratio of the volume of a biconcave $\mathrm{RBC}$ to that of a sphere having a radius equal to the long-axis radius of the $\mathrm{RBC}$.

\section{Relationship between bearing gap size and viscosity}

In this work, $\mathrm{HCT}_{\mathrm{R}}$ values were calculated using Eq. (4). During the various trials, $\mathrm{dx}$ changed along with the hydrodynamic force, which was dependent on the viscosity of the blood. The viscosity, in turn, was primarily determined by the HCT value. In a previous study [17], dx could be measured using a laser displacement meter because the HCT value was quite low (1\%), but this becomes more difficult at higher HCT values. Thus, in the work reported herein, we determined the relationship between $\mathrm{dx}$ and viscosity separately in additional experimental trials that did not use blood. In these experiments, the circuit was made of the same materials and the pump was also operated at $5 \mathrm{~L} / \mathrm{min}$ and 4000 rpm, but a glycerol (Wako Pure Chemical Industries Ltd., Osaka, Japan)/water solution was used as the working fluid. The viscosity of this fluid could be modified by varying the proportion of glycerol and was ascertained with a viscometer (DVM-E-II, Tokimec Inc., Tokyo, Japan). The $\mathrm{dx}$ values were measured using a laser confocal displacement meter with a resolution of $0.2 \mu \mathrm{m}$ (LT8110; Keyence Corp.) and each experiment was conducted three times, with the results as shown in Fig. 2. From these data, it is evident 
Fig. 2 Relationship between the bottom bearing gap, $\mathrm{dx}$, and the viscosity of the working fluid. Each data point represents the average of three replicate experiments

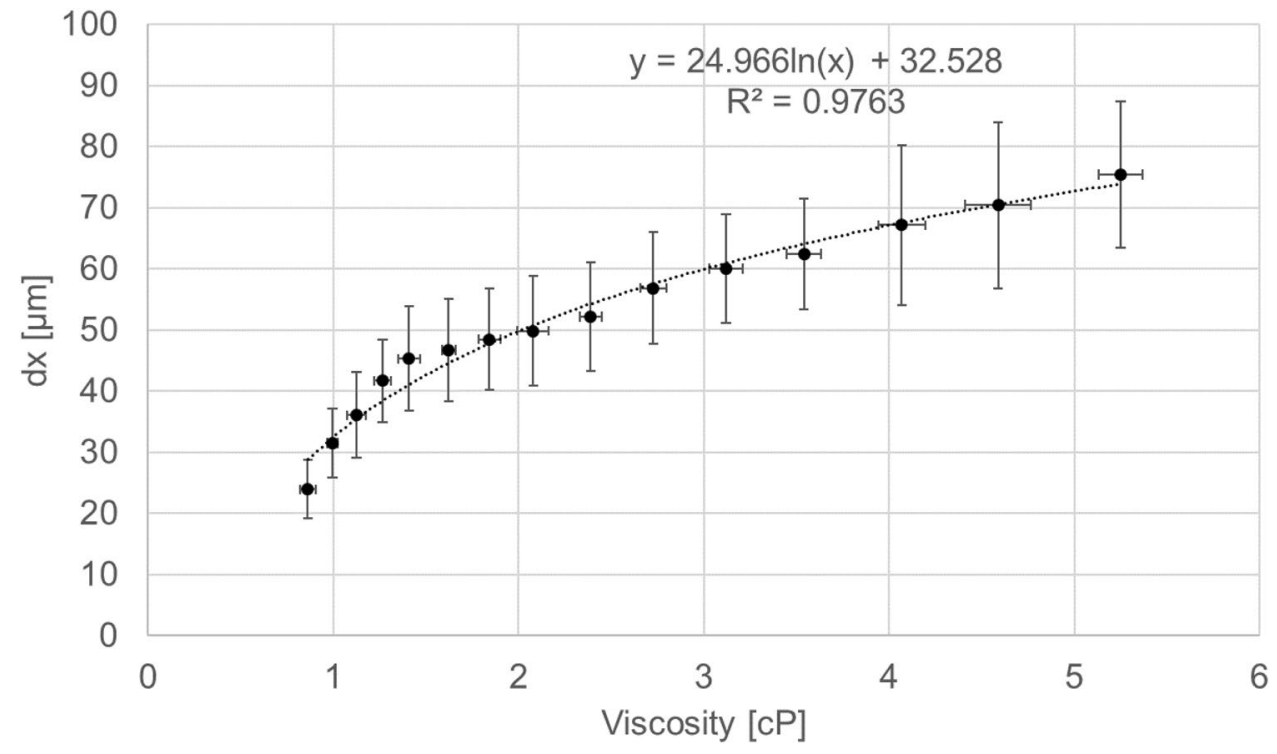

that $d x$ increased as the viscosity was increased. Based on these results, $\mathrm{dx}$ values in the experiments using blood were estimated from the viscosity of the blood at each $\mathrm{HCT}_{\mathrm{W}}$ as measured with a viscometer throughout each trial.

\section{Evaluation of the reliability of plasma skimming efficiency values}

When calculating $\mathrm{HCT}_{\mathrm{R}}$, the $\mathrm{dx}$ at each $\mathrm{HCT}_{\mathrm{W}}$ was obtained by substituting the measured viscosity into the $\mathrm{dx}$-viscosity correlation provided in Fig. 2. However, paradoxically, the viscosity of the blood in the ridge area should equal the viscosity at the $\mathrm{HCT}_{\mathrm{R}}$, and so another index, $\mathrm{PSE}_{\min }(\mathrm{dx}=25 \mu \mathrm{m})$ was also calculated. If PS perfectly occurs, the $\mathrm{HCT}_{\mathrm{R}}$ should be close to zero and the viscosity becomes plasma. Then, since the viscosity of plasma is close to that of water, based on this assumption and Fig. 2, $\mathrm{dx}$ will have a minimum value of approximately $25 \mu \mathrm{m}$ at the viscosity $(0.86 \mathrm{cP})$ of pure water at $37{ }^{\circ} \mathrm{C}$. Based on Eq. 4 , the resulting $\mathrm{HCT}_{\mathrm{R}}$ value will be overestimated when $\mathrm{dx}$ is given a constant minimum value of $25 \mu \mathrm{m}$. Therefore, the use of $\operatorname{PSE}_{\min }(\mathrm{dx}=25 \mu \mathrm{m})$ provides the most conservative estimate of PSE.

It is important to understand the relationship between $Q$ and $d x$ when evaluating the PSE at high HCT values. In the case of $Q=1$, the RBCs occupy only the ridge area, such that the RBC layer will consist of just one RBC that covers the ridge. If $Q=2$, the layer will consist of two RBCs. However, the present system can only evaluate $Q$ over the range of $0 \leq Q \leq 1$ because of the two-dimensional imaging that is employed, as shown in Fig. 1c. The range over which the PSE can be examined can be obtained from the following equation:
$\operatorname{PSE}_{\mathrm{L}}=1-\frac{1}{Q_{\mathrm{W}}}$,

where

$Q_{\mathrm{W}}=\frac{\mathrm{HCT}_{\mathrm{W}} \times d x \times \sigma}{\mathrm{MCV}}$.

$Q_{\mathrm{W}}$ is calculated by substituting $\mathrm{HCT}_{\mathrm{W}}$ for $\mathrm{HCT}_{\mathrm{R}}$ in Eq. (4). Consequently, PSE can be evaluated when $\mathrm{PSE}_{\mathrm{L}} \leq \mathrm{PSE} \leq 1$. It should also be noted that, in the case that $\mathrm{Q}_{\mathrm{W}}<1$, Eq. (6) indicates that $\mathrm{PSE}_{\mathrm{L}}$ will have a value of 0 .

\section{Results}

The mean ( \pm standard deviation) MCV obtained from five replicate trials was $94 \pm 2.7$. The estimated $d x$ values in the $\mathrm{HCT}_{\mathrm{W}}$ whose viscosity in each blood was measured range from 0 to $40 \%$ were approximately $25-60 \mu \mathrm{m}$. The relationship between $\mathrm{dx}$ and $\mathrm{HCT}_{\mathrm{W}}$ was linear. Figure 3 provides typical high-speed photographic images acquired at $\mathrm{HCT}_{\mathrm{W}}$ values from 10 to $35 \%$, from which it is evident that the number of RBCs in the ridge area was decreased as $\mathrm{HCT}_{\mathrm{W}}$ was decreased. The plots in Fig. 3 indicate significant variations in $Q$ with respect to the rotational phase for various $\mathrm{HCT}_{\mathrm{W}}$, so mean values of $Q$ were used for the evaluation of PSE. Mean $Q$ was obtained by averaging $Q$ shown in Fig. 3 over three rotational phases of the impeller. Figure 4 shows the relationship between the mean $Q$ values and $\mathrm{HCT}_{\mathrm{W}}$. By using Fig. 4, PSE and $\mathrm{PSE}_{\min }(\mathrm{dx}=25 \mu \mathrm{m})$ were calculated and the results are shown in Fig. 5a, b, respectively. The mean values for PSE and $\operatorname{PSE}_{\min }(\mathrm{dx}=25 \mu \mathrm{m})$ with respect to $\mathrm{HCT}_{\mathrm{W}}$ are summarized in Table 1. Thus, for $\mathrm{HCT}_{\mathrm{W}}>20 \%$, PSE and $\operatorname{PSE}_{\min }(\mathrm{dx}=25 \mu \mathrm{m})$ both approached $\mathrm{PSE}_{\mathrm{L}}$. 
Fig. 3 Typical high-speed photographic images of the observation point at various $\mathrm{HCT}_{\mathrm{W}}$ and the variations in Q with respect to the impeller rotational phase
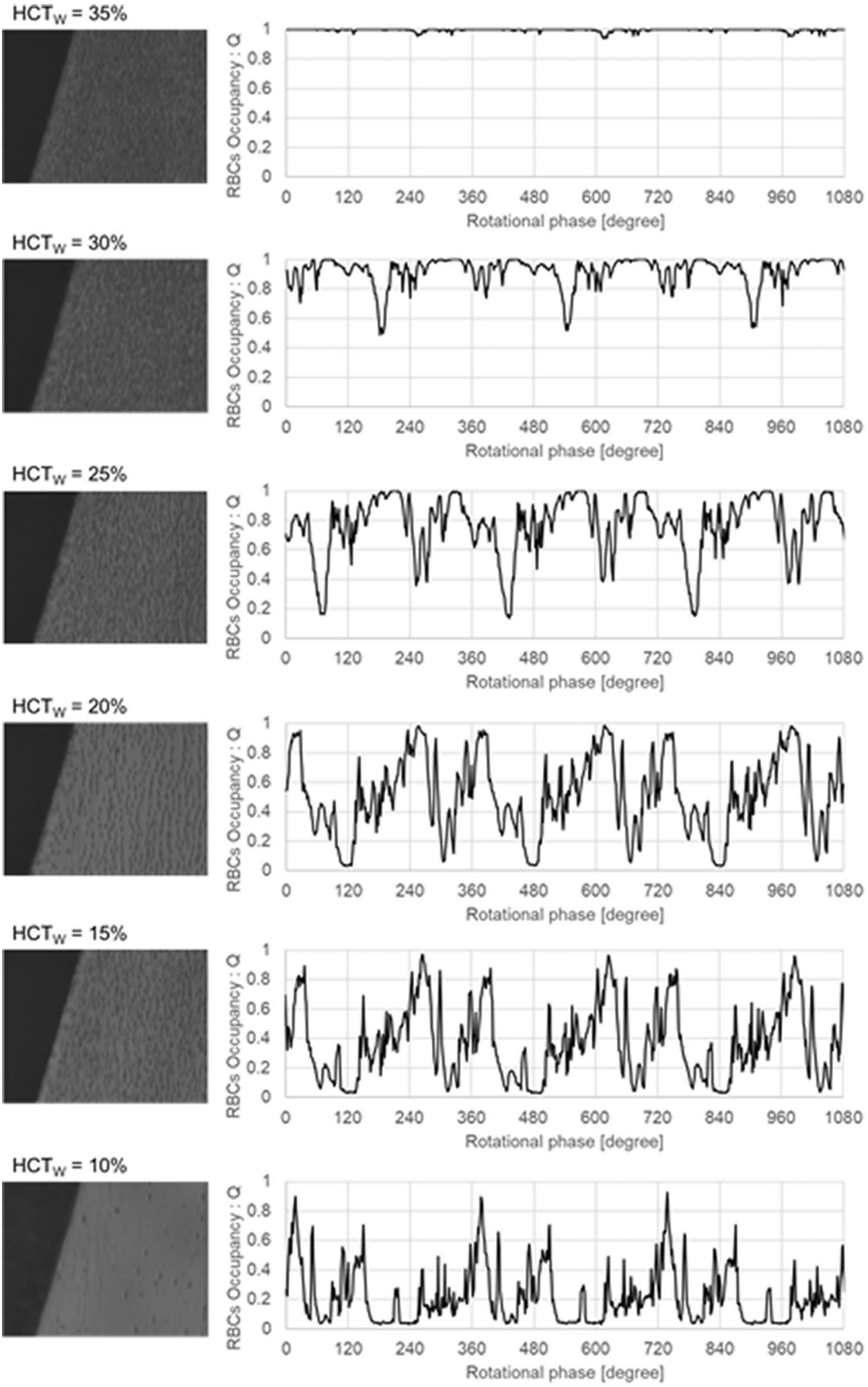

\section{Discussion}

The developed system and the experimental method based on the optical theory of blood demonstrated herein indicate that the PS effect does occur in the SGB of a centrifugal blood pump. The present results show that PS appeared at the PSE was approximately $90 \%$ in over the $\mathrm{HCT}_{\mathrm{W}}$ range from 0 to $20 \%$. The most conservative values, $\mathrm{PSE}_{\min }(\mathrm{dx}=25 \mu \mathrm{m})$, were $75 \%$ or greater, demonstrating that the PS effect occurred with this level of efficiency. In 
Fig. 4 Relationship between the mean $Q$ value obtained by averaging $Q$ shown in this figure over three rotational phases of the impeller and $\mathrm{HCT}_{\mathrm{W}}$

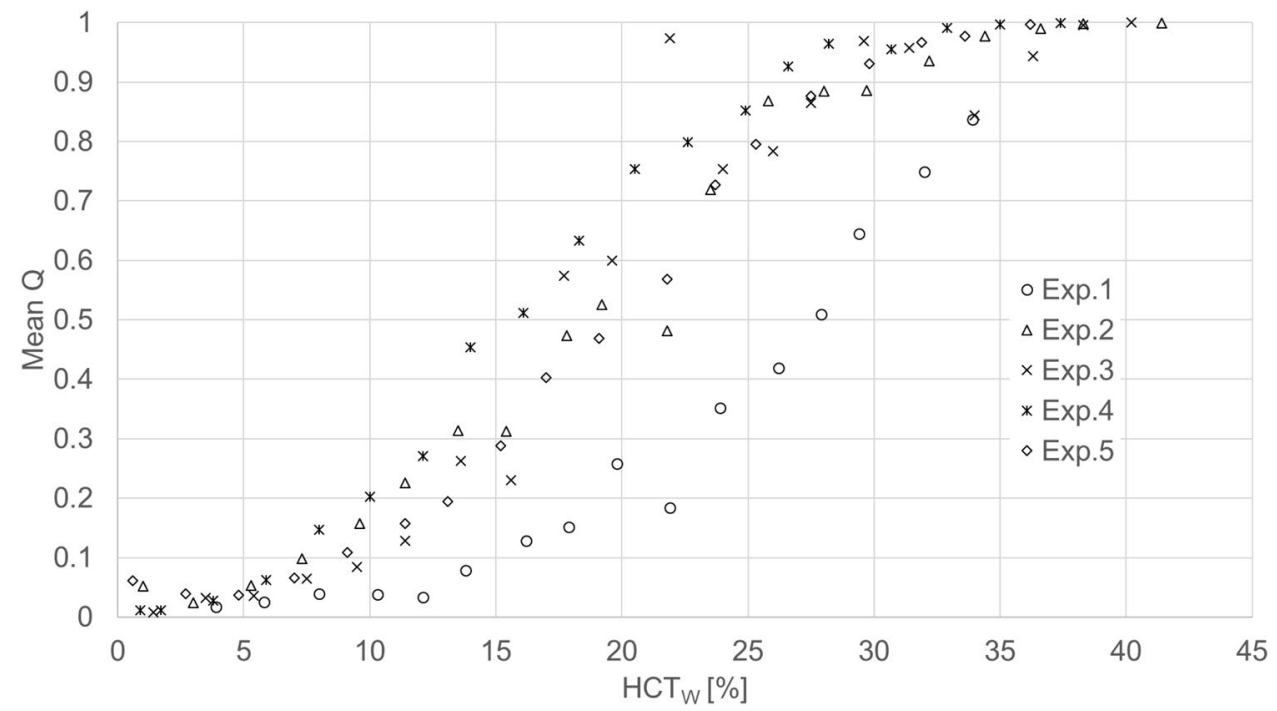

addition, at $\mathrm{HCT}_{\mathrm{W}}$ values less than $20 \%$, each PSE value was significantly higher than $\mathrm{PSE}_{\mathrm{L}}$. Therefore, this study confirmed that a significant level of PS occurred at the $\mathrm{HCT}_{\mathrm{W}}$ values employed. However, above this $\mathrm{HCT}_{\mathrm{W}}$ range, the PSE was essentially equivalent to the $\mathrm{PSE}_{\mathrm{L}}$. The cutoff $\mathrm{HCT}_{\mathrm{W}}$ value for performing an evaluation would therefore be $20 \%$.

\section{Limitations and future work}

The use of a hydrodynamically levitated centrifugal blood pump complicates the study of the PS effect because the bearing gap is affected by the hydrodynamic force. This, in turn, is determined by the rotational speed of the pump and the viscosity of the working fluid. For future study, it is important to investigate the inhibition of hemolysis by PS. However, because the bearing gap changes with increases in the rotational speed, the extent of hemolysis is also increased as the shear stress becomes greater in the gap, and this phenomenon combines with the PS effect. In addition, although a means of estimating $\mathrm{dx}$ when working with blood is proposed in this study, the accuracy of this estimation affects the accuracy with which the PSE value can be obtained. Therefore, it would be desirable to stabilize the gap so that it is not affected by either the impeller rotation or the blood viscosity. It should also be noted that the extent to which the pump used in the present work induced hemolysis increased along with the rotational speed [18]. At present, inhibition of hemolysis by the PS effect is not fully understood. According to Fig. 5a, we speculate that PSE values greater than $90 \%$ might be required before this inhibition becomes significant.

As a conventional approach to reduce the hemolysis of a hydrodynamically levitated centrifugal blood pump, the researchers have tried to develop an SGB to levitate the rotor at a larger gap in order to reduce the shear stress [18]. Although the approach to decrease the bearing gap size would cause plasma skimming, the proposed method would allow ease of observation because the smaller gap size decreases $\mathrm{PSE}_{\mathrm{L}}$, as shown in Eqs. (6) and (7). In the future, the relationship among the gap size, the PSE, and the hemolysis should be investigated.

With regard to theoretical limitations, the optical scattering coefficient, $\mu_{\mathrm{s}}$, in Eq. (2) can be determined in the case that the RBCs are uniformly distributed over the ridge. In contrast, nonuniform distribution will reduce the value of $Q$ because the RBCs may begin to overlap one another, which in turn can cause PSE to be overestimated. However, as shown by the high-speed images in Fig. 3, at $\mathrm{HCT}_{\mathrm{W}}=20$ and $15 \%$, there was a homogeneous dispersion of RBCs over the ridge. In addition, the $\mathrm{PSE}_{\mathrm{L}}$ term in Eq. (6) is the most important limitation when evaluating the PS effect. Thus, the $\mathrm{PSE}_{\mathrm{L}}$ determines whether the PSE can be evaluated, so it is important to examine these variables when assessing the PS effect in the high HCT range.

The limitation reflected in the $\mathrm{PSE}_{\mathrm{L}}$ value originates from the use of two-dimensional imaging, because this type of imaging cannot provide data in the case that $Q$ is larger than 1. It is possible that optical coherence tomography [25-27] might be able to overcome this limitation, because this technique can produce cross-sectional images such as that shown in Fig. 1d. Our own group has also employed a hyperspectral imaging system to monitor thrombus formation in the same pump used in this study [28-31]. This system can generate images based on the optical absorption of hemoglobin in the ridge area by acquiring data at multiple wavelengths, such that the hemoglobin concentration over the ridge can be calculated based on the Beer-Lambert law. As a way not to rely on an optical method to evaluate the PS effect, for an invisible pump, an impedance measurement method of blood may be useful because such a method can measure the local RBC density [32]. In addition, a magnetically levitated 
Fig. 5 a Plasma skimming efficiency, PSE, and the evaluation limit, $\mathrm{PSE}_{\mathrm{L}}$, as functions of $\mathrm{HCT}_{\mathrm{W}} \cdot \mathbf{b}$ Most conservative estimated plasma skimming efficiency, $\operatorname{PSE}_{\min }(\mathrm{dx}=25 \mu \mathrm{m})$, and evaluation limit,

$\operatorname{PSE}_{\mathrm{L}}(\mathrm{dx}=25 \mu \mathrm{m})$, as functions of $\mathrm{HCT}_{\mathrm{W}}$

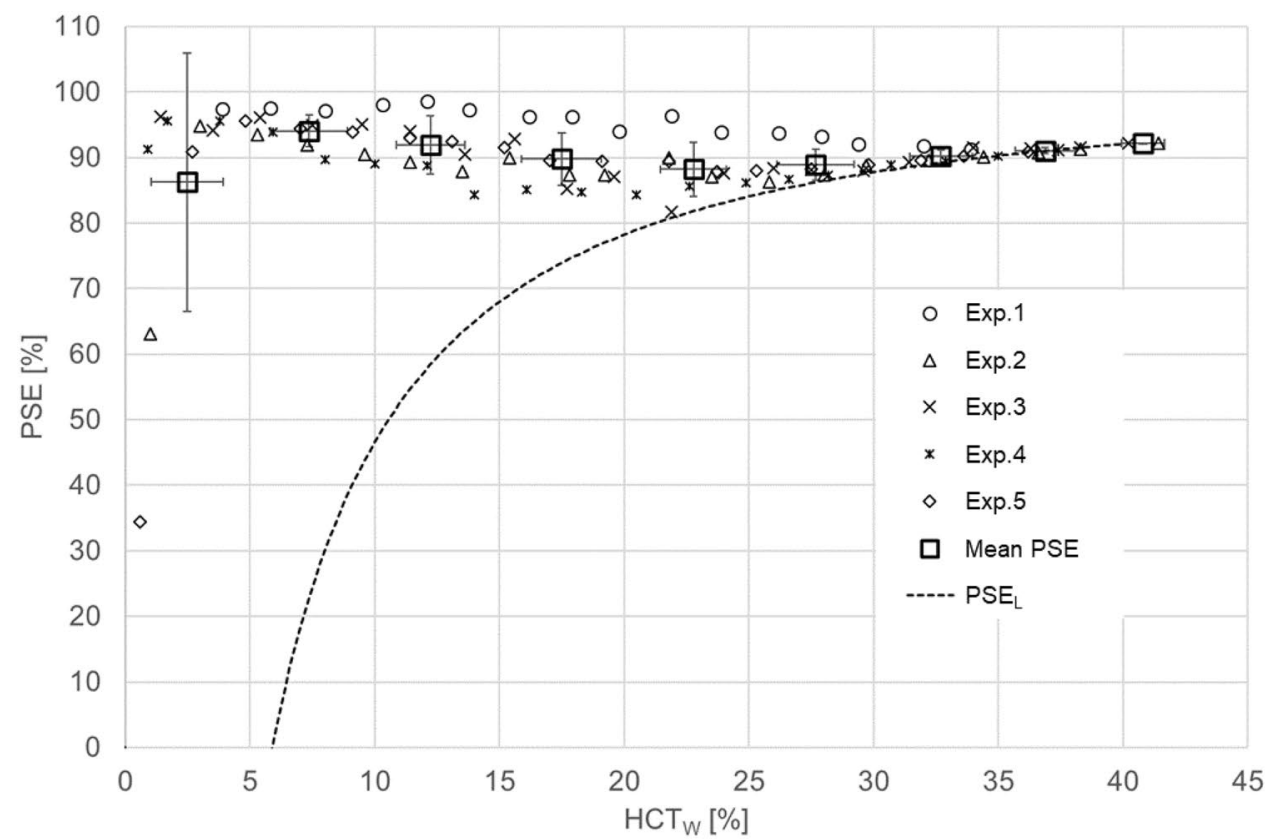

(a)

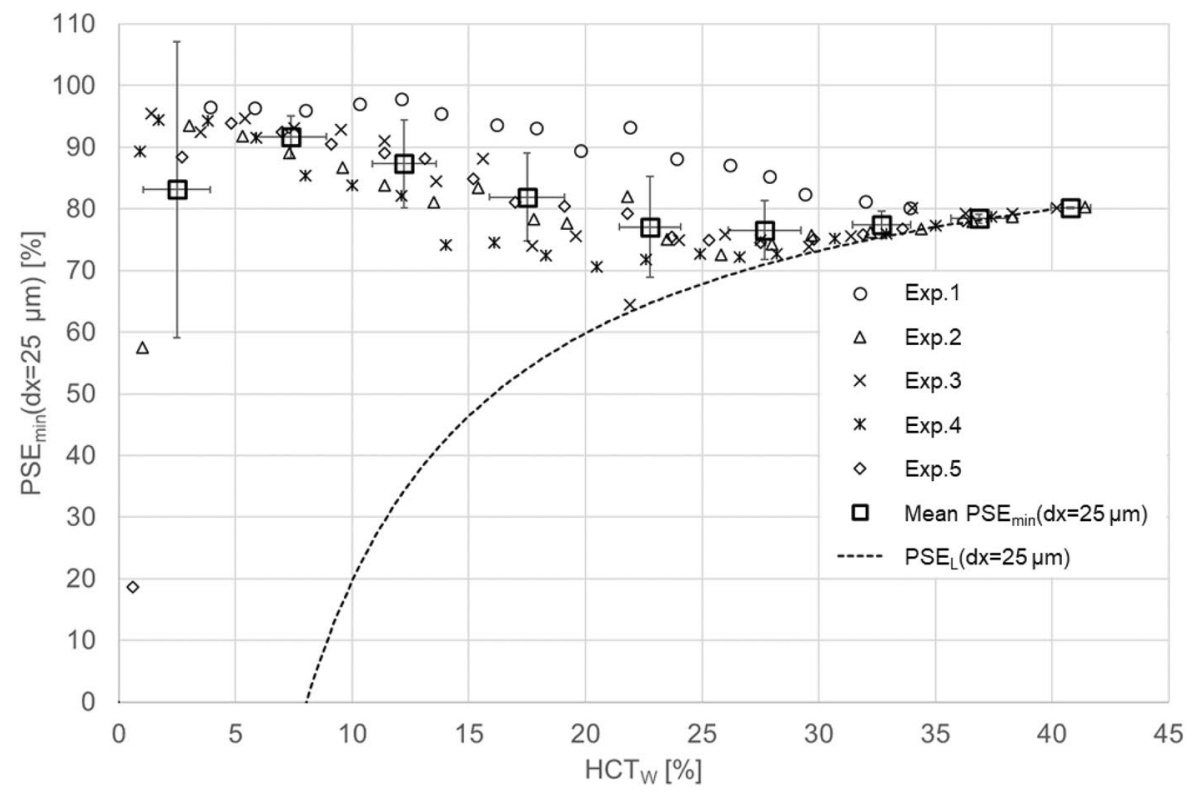

(b) centrifugal blood pump also has potential for the measurement of blood viscosity, which corresponds to $\mathrm{HCT}_{\mathrm{R}}$ [33]. Finally, it is important to note that the imaging of the flow of platelets within an SGB in a rotary blood pump has not yet been achieved. Thus, at present, it is completely unknown if platelets can escape from the ridge area in the same manner as RBCs. Since it is extremely difficult to image platelets under visible or near-infrared light without labeling, fluorescence imaging has typically been employed for this purpose [34-36]. However, the rate of blood flow inside the hydrodynamic bearing of a rotary blood pump is very high, so it will be necessary to development a process allowing the high-speed fluorescent imaging of platelets.

\section{Conclusion}

This work established a method for the limitations associated with evaluating the PSE within an SGB in a hydrodynamically levitated centrifugal blood pump. The results of this study demonstrate that a PS effect occurs in this type of pump in human blood at or below a hematocrit of $20 \%$. We 
Table 1 Mean value ( \pm standard deviation) for PSE and $\mathrm{PSE}_{\min }(\mathrm{dx}=25 \mu \mathrm{m})$ with respect to $\mathrm{HCT}_{\mathrm{W}}$

\begin{tabular}{lll}
\hline $\mathrm{HCT}_{\mathrm{w}}(\%)$ & PSE $(\%)$ & $\begin{array}{l}\mathrm{PSE}_{\text {min }} \\
(\mathrm{dx}=25 \mu \mathrm{m}) \\
(\%)\end{array}$ \\
\hline $2.5 \pm 1.4$ & $86.3 \pm 19.7$ & $83.1 \pm 24.0$ \\
$7.4 \pm 1.5$ & $94.1 \pm 2.4$ & $91.7 \pm 3.4$ \\
$12.2 \pm 1.4$ & $91.9 \pm 4.5$ & $87.3 \pm 7.1$ \\
$17.5 \pm 1.6$ & $89.8 \pm 3.9$ & $81.9 \pm 7.1$ \\
$22.8 \pm 1.3$ & $88.2 \pm 4.2$ & $77.1 \pm 8.2$ \\
$27.7 \pm 1.5$ & $88.9 \pm 2.4$ & $76.5 \pm 4.8$ \\
$32.7 \pm 1.2$ & $90.2 \pm 1.0$ & $77.4 \pm 2.2$ \\
$36.9 \pm 1.2$ & $91.0 \pm 0.5$ & $78.5 \pm 0.7$ \\
$40.8 \pm 0.8$ & $92.0 \pm 0.0$ & $80.2 \pm 0.1$ \\
\hline
\end{tabular}

The number of experiments is five times

believe that the PS effect in a rotary blood pump should be investigated in more detail to permit future advances, such as ultra-miniaturization or hemocompatibility, and the method proposed herein should assist in such research.

Acknowledgements The present study was supported by JSPS KAKENHI Grant number JP16K01444.

\section{Compliance with ethical standards}

Conflict of interest The authors declare that there is no conflict of interest in respect of this study.

Open Access This article is licensed under a Creative Commons Attribution 4.0 International License, which permits use, sharing, adaptation, distribution and reproduction in any medium or format, as long as you give appropriate credit to the original author(s) and the source, provide a link to the Creative Commons licence, and indicate if changes were made. The images or other third party material in this article are included in the article's Creative Commons licence, unless indicated otherwise in a credit line to the material. If material is not included in the article's Creative Commons licence and your intended use is not permitted by statutory regulation or exceeds the permitted use, you will need to obtain permission directly from the copyright holder. To view a copy of this licence, visit http://creativecommons.org/licenses/by/4.0/.

\section{References}

1. Burgreen GW, Antaki JF, Wu ZJ, Holmes AJ. Computational fluid dynamics as a development tool for rotary blood pumps. Artif Organs. 2002;25:336-40.

2. Apel J, Paul R, Klaus S, Siess T, Reul H. Assessment of hemolysis related quantities in a microaxial blood pump by computational fluid dynamics. Artif Organs. 2002;25:341-7.

3. Su B, Chua LP, Wang X. Validation of an axial flow blood pump: computational fluid dynamics results using particle image velocimetry. Artif Organs. 2012;36:359-67.

4. Yang X, Gui X, Huang H, Shen Y, Yu Z, Zhang Y. Particle image velocimetry experimental and computational investigation of a blood pump. J Therm Sci. 2012;21:262-8.
5. Fraser KH, Zhang T, Taskin ME, Griffith BP, Wu ZJ. A quantitative comparison of mechanical blood damage parameters in rotary ventricular assist devices: shear stress, exposure time and hemolysis index. J Biomech Eng. 2012;134:081002. https://doi. org/10.1115/1.4007092.

6. Krogh A. Studies on the physiology of capillaries: II. The reactions to local stimuli of the blood-vessels in the skin and web of the frog. J Physiol. 1921;55:412-22.

7. Palmer AA. Axial drift of cells and partial plasma skimming in blood flowing through glass slits. Am J Physiol. 1965;209:1115-22.

8. Perkkiö J, Keskinen R. Hematocrit reduction in bifurcations due to plasma skimming. Bull Math Biol. 1983;45:41-50.

9. Yang J, Yoo SS, Lee TR. Effect of fractional blood flow on plasma skimming in the microvasculature. Phys Rev E. 2017;95:040401.

10. Enden G, Popel AS. A numerical study of plasma skimming in small vascular bifurcations. J Biomed Eng. 1994;116:79-88.

11. Lee TR, Yoo SS, Yang J. Generalized plasma skimming model for cells and drug carriers in the microvasculature. Biomech Model Mechanobiol. 2017;16:497-507.

12. Lewandowski JJ, Malchesky PS, Pentermann EJ, Breuer AC, Lynn M, Nose Y. Flow visualization of blood at the wall of a porous fiber. Trans Am Soc Artif Intern Organs. 1985;31:419-23.

13. Kink T, Reul H. Concept for a new hydrodynamic blood bearing for miniature blood pumps. Artif Organs. 2004;28:916-20.

14. Amaral F, Egger C, Steinseifer U, Schmiz-Rode T. Differences between blood and a Newtonian fluid on the performance of a hydrodynamic bearing for rotary blood pumps. Artif Organs. 2013;37:786-92.

15. Leslie LJ, Marshall LJ, Devitt A, Hilton A, Tansley GD. Cell exclusion in Couette flow: evaluation through flow visualization and mechanical forces. Artif Organs. 2013;37:267-75.

16. James NL, Wilkinson CM, Lingard NL, van der Meer AL, Woodard JC. Evaluation of hemolysis in the VentrAssist implantable rotary blood pumps. Artif Organs. 2003;27:108-13.

17. Murashige T, Sakota D, Kosaka R, et al. Plasma skimming in a spiral groove bearing of a centrifugal blood pump. Artif Organs. 2016;40:856-66.

18. Murashige T, Kosaka R, Sakota D, et al. Evaluation of a spiral groove geometry for improvement of hemolysis level in a hydrodynamically levitated centrifugal blood pump. Artif Organs. 2015;39:710-4.

19. Yaroslavsky AN, Priezzhev AV, Rodriguez J, Yaroslavsky IV, Battarbee H. Optics of blood. In: Tuchin VV, editor. Handbook of optical biomedical diagnostics. Washington: SPIE Press; 2002. p. 169-216.

20. Twersky V. Absorption and multiple scattering by biological suspensions. J Opt Soc Am. 1970;60:1084-93.

21. Roggan A, Friebel M, Doershel K, Hahn A, Mueller G. Optical properties of circulating human blood in the wavelength range 400-2500 nm. J Biomed Opt. 1999;4:36-46.

22. Ishimaru A. Wave propagation and scattering in random media. New York: Academic Press; 1978.

23. Steinke JM, Shepherd AP. Role of light scattering in whole blood oximetry. IEEE Trans Biomed Eng. 1986;33:294-301.

24. Sakota D, Takatani S. Photon-cell interactive Monte Carlo model based on the geometric optics theory for photon migration in blood by incorporating both extra- and intracellular pathways. J Biomed Opt. 2010;15:065001.

25. Matsuhashi Y, Sameshima K, Yamamoto Y, Umezu M, Iwasaki $\mathrm{K}$. Real-time visualization of thrombus formation at the interface between connectors and tubes in medical devices by using optical coherence tomography. PLoS ONE. 2017a;12:e0188729.

26. Matsuhashi Y, Sameshima K, Yamamoto Y, Umezu M, Iwasaki $\mathrm{K}$. Investigation of the influence of fluid dynamics on thrombus 
growth at the interface between a connector and tube. J Artif Organs. 2017b;20:293-302.

27. Chen CL, Wang RK. Optical coherence tomography based angiography. Biomed Opt Express. 2017;8:1056-82.

28. Sakota D, Murashige T, Kosaka R, Nishida M, Maruyama O. Feasibility of the optical imaging of thrombus formation in a rotary blood pump by near-infrared light. Artif Organs. 2014;38:733-40.

29. Sakota D, Murashige T, Kosaka R, Fujiwara T, Nishida M, Maruyama O. Real-time observation of thrombus growth process in an impeller of a hydrodynamically levitated centrifugal blood pump by near-infrared hyperspectral imaging. Artif Organs. 2015;39:714-9.

30. Sakota D, Murashige T, Kosaka R, Fujiwara T, Nishida M, Maruyama O. Noninvasive optical imaging of thrombus formation in mechanical circulatory support devices. J Biorheol. 2016;30:6-12.

31. Fujiwara T, Sakota D, Ohuchi K, et al. Optical dynamic analysis of thrombus inside a centrifugal blood pump during extracorporeal mechanical circulatory support in a porcine model. Artif Organs. 2017;41:893-903.

32. Li J, Sapkota A, Kikuchi D, Sakota D, Maruyama O, Takei M. Red blood cells aggregability measurement of coagulating blood in extracorporeal circulation system with multiple-frequency electrical impedance spectroscopy. Biosens Bioelectron. 2018;112:79-85.

33. Hijikata W, Rao J, Abe S, Takatani S, Shinshi T. Sensorless viscosity measurement in a magnetically-levitated rotary blood pump. Artif Organs. 2015;39:559-68.

34. Ruggeri ZM, Orje JN, Habermann R, Federici AB, Reininger AJ. Activation-independent platelet adhesion and aggregation under elevated shear stress. Blood. 2006;108:1903-10.

35. Schulze H, Stegner D. Imaging platelet biogenesis in vivo. Res Pract Thromb Haemost. 2018;2:461-8.

36. Mizuno M, Tomizawa A, Ohno K, Jakubowski JA, Sugidachi A. A novel model of intravital platelet imaging using CD41-ZsGreen1 transgenic rats. PLoS ONE. 2016;11:e0154661.

Publisher's Note Springer Nature remains neutral with regard to jurisdictional claims in published maps and institutional affiliations. 